Journal of Animal and Veterinary Advances 9 (21): 2679-2684, 2010

ISSN: $1680-5593$

(C) Medwell Journals, 2010

\title{
The Polyclonal Antibody Against Chicken Interleukin-6 Prepared by Immunization of Recombinant Eukaryotic Plasmid Can React Efficiently with its Prokaryotic Expression Protein
}

\author{
${ }^{1}$ Min Li, ${ }^{1}$ Yuanping Wang, ${ }^{1}$ Nianli Zou, ${ }^{1}$ Sanjie Cao, ${ }^{1}$ Xintian Wen and ${ }^{1,2}$ Yong Huang \\ ${ }^{1}$ College of Veterinary Medicine, \\ ${ }^{2}$ Key Laboratory of Animal Disease and Human Health of Sichuan Province, \\ Sichuan Agricultural University, Ya'an 625014, China
}

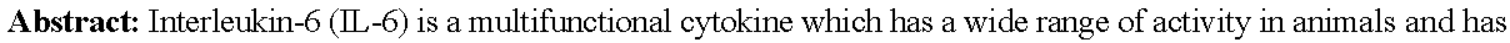
an impact on almost all immune system cells. To produce specific antibody against chicken IL- 6 and detect its activity, its complete Open Reading Frame (ORF) sequence was amplified and inserted into the eukaryotic expression vector pcDNA3.1 ( + ) to get recombinant eukaryotic plasmid pcDNA-ChIL-6 and BALB/c mouse were immunized with this plasmid to produce polyclonal antibody against Chicken IL-6. Then the mature protein coding genes of chicken IL- 6 was cloned and inserted into the prokaryotic expression vector pET-32a (+) to get recombinant prokaryotic plasmid pET-ChLL-6, the expression of this fusion protein was successfully induced by Isopropy $1-\beta-D$-thiogalactoside (IPTG) and the recombinant protein was further purified by NTA His•Bind column. The purified recombinant protein could react positively with polyclonal antibody against pcDNA-ChIL6 in Western-blot and indirect ELISA.
\end{abstract}

Key words: Chicken IL-6, eukaryotic expression, prokaryotic expression, polyclonal antibody, Western-blot, indirect ELISA

\section{INTRODUCTION}

Cytokines are small-molecule soluble regulatory peptide which secreted by immune cells. In the immune response, cytokines play an important regulatory role in the interaction between cells as well as modulating inflammatory responses (Poquan, 2001).

Interleukin-6 plays roles in regulating immune responses, acute phase reactions and haematopoiesis inducing proliferation and antibody production in hybridoma cells (Kishimoto et al., 1995) and has the potential to become vaccine molecular adjuvant and alternatives to antibiotics (Hilton et al., 2002). IL-6 is produced by many types of cells and can act on B cells (Hirano et al., 1986), T cells (Houssiau et al., 1988), hepatocytes (Gauldie et al., 1987), haematopoietic progenitor cells (Ikebuchi et al., 1987) and cells of the central nervous system (Satoh et al., 1988).

In the immune system, $\mathbb{L}-6$ can induce differentiation of activated $\mathrm{B}$ cells to antibody producing cells and the activation, growth and differentiation of T-cell (Muraguchi et al., 1988; Van Snick, 1990). It was also demonstrated to induce interferon- $\alpha(\mathrm{IFN}-\alpha)$ in spleens of chickens (Karaca et al., 1996; Sick et al., 1998). The complete open reading frame sequence of chicken IL-6 was cloned by Scheider (Schneider et al., 2001) from spleens of chickens using suppression subtractive hybridization technology.

In this study, chicken IL-6 (ChIL-6) gene was cloned and expressed and the polyclonal antibody was obtained by inoculating $\mathrm{BALB} / \mathrm{c}$ mice with eukaryotic plasmid DNA of chicken IL-6 gene. The mature protein of ChIL- 6 was also expressed in $E$. coli. The activity of this polyclonal antibody was further verified by Western-blot and Indirect-ELISA.

\section{MATERIALS AND METHODS}

Bacterial trains, vector and grown conditions: Escherichia coli DH5 $\alpha$ and BL21 (DE3) were purchased from invitrogen corporation (Madison, California, USA). The T-cloning vector $\mathrm{pMD} 18$-T plasmid were purchased from Takara biotechnology (Dalian, China) co., ltd, Plasmid pcDNA3.1 $(+)$ was purchased from invitrogen corporation (California, USA) and PET-32a $(+)$ was purchased from Novagen (Darmstadt, Germany). E. coli was grown at $37^{\circ} \mathrm{C}$ in Luria-Bertani broth or on Luria-Bertani broth agar supplemented when necessary with ampicillin $\left(50 \mu \mathrm{g} \mathrm{mL}^{-1}\right)$ for selection and maintenance of recombinant plasmids.

Corresponding Author: Yong Huang, College of Veterinary Medicine, Sichuan Agricultural University, Ya'an 625014, China 
Cloning of ChIL-6 and constructions of eukaryotic expression vector: About 4 weeks old chickens of Ya'an local species were killed and spleen was removed then the spleen was ground immediately and splenic lymphocyte were isolated by utilizing lymphocyte separation medium (Haoyang Biological Manufacture Co., Ltd. Tianjin, China) following the manufacturer's instructions. The splenocytes lymphocytes were stimulated with ConA (10 $\mathrm{g} \mathrm{mL}^{-1}$ ) for $20 \mathrm{~h}$ and total RNA was extracted using Trizol reagent (Takara biotechnology, Dalian, China) according to the manufacturer's protocol. Purified RNA was resuspended in $30 \mu \mathrm{L}$ RNase-free water and stored at $-70^{\circ} \mathrm{C}$ until further use.

For the Reverse Transcription (RT) reaction, the Quantscript RT Kit (TIANGEN Biotech Co., LTD, Beijing, China) was used and the total reaction volume was $40 \mu \mathrm{L}$, including $4 \mu \mathrm{L}$ of $10 \times \mathrm{RT}$ buffer, $4 \mu \mathrm{L}$ of random 6 mers $(10 \mu \mathrm{M}), 4 \mu \mathrm{L}$ of dNTP mix (2.5 mM each), $2 \mu \mathrm{L}$ of Quant Reverse Transcriptase, $20 \mu \mathrm{L}$ of template RNA and $6 \mu \mathrm{L}$ Rnase-free water. The reaction mixture was incubated at $37^{\circ} \mathrm{C}$ for $60 \mathrm{~min}$ and $94^{\circ} \mathrm{C}$ for $4 \mathrm{~min}$. Then $5 \mu \mathrm{L}$ of the RT product was used to amplify chicken IL-6 using the following primers: P1: F5' GCGTCGACTCAGGCACTG AAACTCCTGG 3 ' with XhoI site (underlined); P2: GCGTC GACTCAGGCACTGAAACTCCTGG $3^{\prime}$ with Sal I site (underlined). Amplification of the DNA was performed using $2 \times$ taq PCR mixture $25 \mu \mathrm{L}$ (TIANGEN, Beijing), forward primer $2.0 \mu \mathrm{L}$, reverse primer $2.0 \mu \mathrm{L}$, template DNA $4.0 \mu \mathrm{L}, 17 \mu \mathrm{L}$ water comprising a total volume of $50 \mu \mathrm{L}$. The optimum conditions for PCR were as follows: $94^{\circ} \mathrm{C}$ for $4 \mathrm{~min}, 30$ cycles at $94^{\circ} \mathrm{C}$ for $30 \mathrm{sec}, 53^{\circ} \mathrm{C}$ for $30 \mathrm{sec}, 72^{\circ} \mathrm{C}$ for $40 \mathrm{sec}$ and final elongation at $72^{\circ} \mathrm{C}$ for $10 \mathrm{~min}$. The PCR product was analyzed in $0.9 \%$ agarose and extracted from the gels with E.N.Z.A.1 Gel extraction kit (Omega, USA) following the manufacturer's instruction. The PCR product (about $730 \mathrm{bp}$ ) was ligated with PMD18-T vector and then transformed into $E$. coli DH $5 \alpha$. Confirmation of clones containing recombinant plasmid was achieved by PCR and Restriction Enzyme (RE) digestion. The correct clones were sequenced by Sangon (Shanghai, China) co., Ltd.

Both the recombinant pMD18-T-ChIL-6 and pcDNA3.1 ( + ) were digested with Xho I and Sal I, then ligated with T4 DNA ligase to yield eukaryotic expression plasmid pcDNA-ChIL-6. Confirmation of clones containing recombinant plasmid was achieved by PCR and RE digestion.

Production of polyclonal antibody against chicken IL-6: After large-scale isolation of recombinant eukaryotic plasmid pcDNA-ChIL-6 DNA using plasmid Maxi kit (OMEGA) following the manufacturer's instructions. The concentration of plasmids was determined by Smartspec
Plus spectrophotometer (BIO-RAD). Twelve BALB/c mice obtained from (the experimental animal center of Sichuan University (Chengdu, China) were injected intramuscularly with $200 \mu \mathrm{L}$ Tris-EDTA buffer (TE) containing $200 \mu \mathrm{g}$ of pcDNA-ChIL-6 on days 0,14 and 28 , respectively. Another twelve mice received $200 \mu \mathrm{g}$ of the pcDNA3.1 ( + . Mice were bled from eyes 10 days following the final boost and sera were collected.

Construction of recombinant prokaryotic expression vector: A pair of specific primers was designed to amplify the mature peptide of $\mathrm{ChLL}-6$ gene. The primers are given as follows: 5' CATGGATCCCCGCTGCCCGCCGCC 3' with BamH I site (underlined); 5' AGCTCGAGTCTCAGGCAC TGAAACTCC 3 ' with Xho I site (underlined). Amplification of the DNA was performed following the procedure above except the template DNA was pcDNA-ChIL-6. Amplification of the DNA was performed using $2 \times$ taq PCR mixture $25 \mu \mathrm{L}$ (TIANGEN, Beijing), forward primer $2.0 \mu \mathrm{L}$, reverse primer $2.0 \mu \mathrm{L}$, template DNA $1.0 \mu \mathrm{L}, 20 \mu \mathrm{L}$ water comprising a total volume of $50 \mu \mathrm{L}$. The optimum conditions for PCR were as follows: $94^{\circ} \mathrm{C}$ for $4 \mathrm{~min}, 30$ cycles at $94^{\circ} \mathrm{C}$ for $30 \mathrm{sec}, 60^{\circ} \mathrm{C}$ for $30 \mathrm{sec}$, $72^{\circ} \mathrm{C}$ for $30 \mathrm{sec}$ and final elongation at $72^{\circ} \mathrm{C}$ for $10 \mathrm{~min}$. The PCR product (about $603 \mathrm{bp}$ ) was inserted into PMD18-T vector and then introduced into $E$. coli $\mathrm{DH} 5 \alpha$. The recombinant plasmid was sequenced by Sangon (Shanghai, China). The recombinant pMD18-T plasmid containing the target gene and $\mathrm{pET}-32 \mathrm{a}(+)$ were digested with BamH I and XhoI, then ligated with T4 DNA ligase. The ligated products were transformed into $E$. coli $\mathrm{DH} 5 \alpha$ and then transformed into BL21 to generate recombinant prokaryotic expression plasmid pET-ChI-6. Confirmation of clones containing recombinant plasmid was achieved by PCR and RE digestion.

Expression and purification of recombinant protein: The BL21 strains containing the recombinant prokaryotic plasmid were grown overnight with shaking at $37^{\circ} \mathrm{C}$. The overnight cultures were diluted in the proportion of $1: 100$ and grown at $37^{\circ} \mathrm{C}$ with vigorous shaking for $3-4 \mathrm{~h}$ until the optical density (OD600) of the cultured cells reached 0.6 , expression of the fusion protein was induced with $1 \mathrm{mM}$ IPTG for $4 \mathrm{~h}$ at $37^{\circ} \mathrm{C}$ (Zou et al., 2010). The cells of $1.0 \mathrm{~mL}$ were harvested by centrifugation at $8000 \mathrm{rpm}$ for $5 \mathrm{~min}$ and washed once with $1 \mathrm{~mL}$ distilled water at $8000 \mathrm{rpm}$ for $5 \mathrm{~min}$.

Then cells were resuspended with $100 \mu \mathrm{L}$ distilled water and $25 \mu \mathrm{L} 5 \times \mathrm{SDS}$ loading buffer after heating at $100^{\circ} \mathrm{C}$ for $5 \mathrm{~min}$, recombinant protein was analyzed by SDS-PAGE. For purification of recombinant ChIL-6, the strains were harvested by centrifugation and then 
suspended in Phosphate-Buffered Saline (PBS) ( $\mathrm{pH} 8.0$ ). After repeated freezing and thawing for 3 times, the suspension were sonicated, centrifuged and resuspended with PBS containing $6 \mathrm{~mol} \mathrm{~L}^{-1}$ guanidine- $\mathrm{HCl}$ for overnight at $4^{\circ} \mathrm{C}$. After centrifugation at $10000 \mathrm{rpm}$ for $60 \mathrm{~min}$, the supernatant was filtrated through $0.45 \mu \mathrm{m}$ filtration membrane.

Then the solution was purified on a column packed with Ni-NTA His•Bind superflow according to the manufacture's instruction (Merck, Darmstadt, Germany). The eluate from each imidazole concentration were collected and analyzed by SDS-PAGE and then treated with different concentrations of guanidine- $\mathrm{HCl}$ (from 5-0 M) in renaturation buffer (50 mM Tris-Hcl (PH8.0) (0.1) $\mathrm{mM}$ oxidized glutathione( $1 \mathrm{mM}$ reduced glutathione) for $24 \mathrm{~h}$ at $4^{\circ} \mathrm{C}$, respectively.

Western blot: The purified proteins were separated by SDS-PAGE gels and then transferred to Polyvinylidene Difluoride (PVDF) membrane with $0.45 \mu \mathrm{m}$ pore size (Millipore Corp., USA) at $15 \mathrm{~V}$ for $1.5 \mathrm{~h}$ using a Transblot Cell (Bio-Rad) according to the manufacturer's instructions. To prevent non-specific binding of antibodies, the membrane was blocked for 60 min with milk buffer (20 mM Tris- $\mathrm{HCl} \mathrm{pH} 8.0,150 \mathrm{mM} \mathrm{NaCl}, 0.05 \%$ Tween $20,5 \%$ skimmed dry milk) at $37^{\circ} \mathrm{C}$. Then the membranes were washed by tris-buffered saline with Tween 20 (TBST) buffer (100 mM Tris- $\mathrm{HCl}$ pH8.0, $150 \mathrm{mM}$ $\mathrm{NaCl}, 0.05 \%$ Tween 20 ) for 3 times and incubated with mouse anti-ChIL-6 polyclonal antibody diluted 1:100 in $0.5 \%$ Bovine Serum Albumin (BSA)/PBS for $60 \mathrm{~min}$ at $37^{\circ} \mathrm{C}$. After washed by TBST for three times, the membrane was incubated with a HRP-labeled sheep anti-mouse IgG (zhongshan Goldenbridge Biotechnology Co., LTD, Beijing, China) for $60 \mathrm{~min}$ at $37^{\circ} \mathrm{C}$. After washed by TBST for three times, target proteins were visualized using 3,3'-diaminobenzidine (DAB) (Tiangen, Beijing, China).

Polyclonal antibody titer analysis by ELISA: Antibody titer was measured using an indirect Enzyme Linked Immunosorbent Analysis (ELISA). Checker board method was used to determine the optimal antigen coating concentration. The purified recombinant antigen diluted to $0.025 \mathrm{~g} \mathrm{~mL}^{-1}$ in carbonate solution ( $\mathrm{pH}$ 9.6) was coated on 96 well microtiter plate (Costar, USA) at $100 \mu \mathrm{L}$ per well incubated at $4^{\circ} \mathrm{C}$ overnight.

The wells were then washed three times by PBST buffer $(0.05 \%$ Tween 20 in PBS) and blocked for $60 \mathrm{~min}$ with $5 \%$ milk buffer at $37^{\circ} \mathrm{C}$. After washed, the wells were incubated with $100 \mu \mathrm{L}$ mouse anti-ChIL-6 polyclonal antibody at different dilutions (from 1:40-
$1: 5120$ ) and incubated at $37^{\circ} \mathrm{C}$ for $60 \mathrm{~min}$. Then the wells were washed and incubated with $100 \mu \mathrm{L}$ of sheep antimouse IgG diluted $1: 5000$ in $0.1 \% \mathrm{BSA} / \mathrm{PBS}$ at $37^{\circ} \mathrm{C}$ for 60 min washed again and detected with $50 \mu \mathrm{L}$ of $3,3^{\prime}, 5,5^{\prime}-$ Tetramethyl Benzidine (TMB) for $10 \mathrm{~min}$ at room temperature. The reaction was stopped by the addition of $35 \mu \mathrm{L}$ of $2 \mathrm{M} \mathrm{H}_{2} \mathrm{SO}_{4}$. The Optical Density (OD) value was read at $450 \mathrm{~nm}$ using a Bio-Rad model 860 plate reader (Bio-Rad, CA, USA) and mouse anti-pcDNA3.1 (+) were added.

For determine the cut-off value, twenty sera samples from mice infected with PBS were used as control group sera, the cut-off value was calculated using the formula: mean of the negative serum values plus three Standard Deviations (SDs).

\section{RESULTS}

Cloning of ChIL-6 and constructions of eukaryotic expression vector: The ChIL- 6 cDNA was acquired by RT-PCR from splenocytes lymphocytes of chicken. The PCR products were analyzed on $1 \%$ agarose gel electrophoresis then one band of 734 bp DNA fragment was observed (Fig. 1). Based on the sequencing result, the ORF of ChIL- 6 was 726 bp long, containing 241 amino acid residues and having a signal peptide of 47 amino

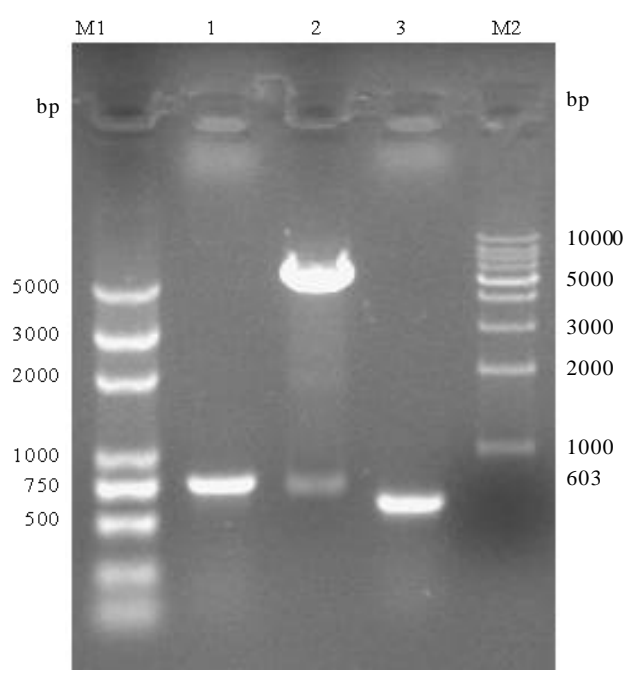

Fig. 1: RT-PCR result of ChIL-6 and Identification of ChIL-6 eukaryotic expression vector Lane M1: DL5000 DNA Marker. Lane 1: RT-PCR result of ChIL-6 complete reading frame gene. Lane 2: Products from pcDNA-ChIL- 6 digested by XhoI and SalI. Lane 3: PCR result of ChIL-6 mature protein encoding gene. Lane M2: DL10000 DNA Marker 


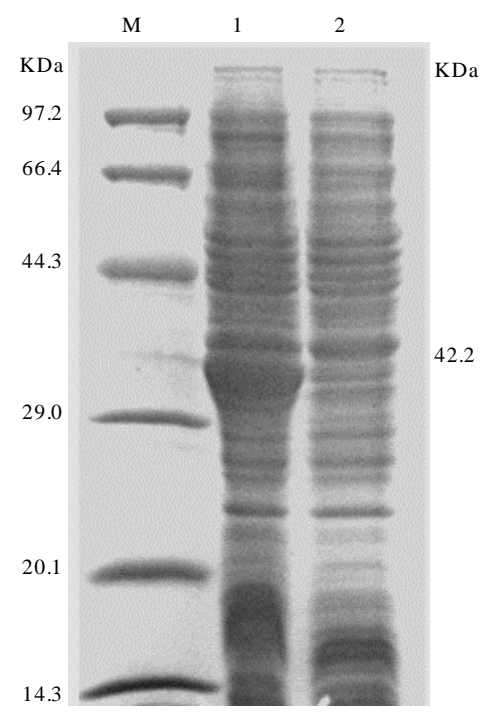

Fig. 2: SDS-PAGE results of expression of recombinant protein, Lane M: Protein marker, Lane 1: Induced BL21 stains containing recombinant plasmid for 4 h. Lane 2: Non-induced BL21 stains

acids. The nucleotide sequence has been sent to the GenBank (accession number: HM367074). The BLAST network server of the National Center for Biotechnology Information (NCBI) were used to compare the homology of chicken $\mathbb{L}-6$ genes in GenBank, the results showed that the nucleotide sequence identities between sequence HM367074 and other four chicken IL-6 genes NM204628.1, AB191038.1, EU170468.1, AB302327.1 were $99.0,99.0,98.0$ and $98.0 \%$, respectively. No significant similarity was found between sequence of chicken IL- 6 and the human IL- 6 genes. The complete ORF of chicken IL-6 was cloned into pMD-18 and then pCDNA3.1, RE analysis showed that eukaryotic expression plasmid pcDNA-ChIL-6 was successfully constructed (Fig. 1).

\section{Construction of recombinant prokaryotic expression} vector, expression and purification of $\mathrm{ChIL}-6$ protein: The mature peptide ChIL-6 gene of $603 \mathrm{bp}$ long was amplified by PCR (Fig. 1) and cloned into pET-32a (+) to achieve the recombinant prokaryotic plasmid PET-32ChIL-6. The PET-32-ChIL-6 was expressed to produce a fusion protein band of approximately $42 \mathrm{kDa}$ size by SDS-PAGE (Fig. 2). The fusion protein was purified using NTA His•Bind column and the optimal wash concentration of imidazole was $100 \mathrm{mM}$. The purified protein showed a single target band as shown by SDS-PAGE (Fig. 3). The concentration of purified protein reached $1.5 \mathrm{mg} \mathrm{mL}^{-1}$.

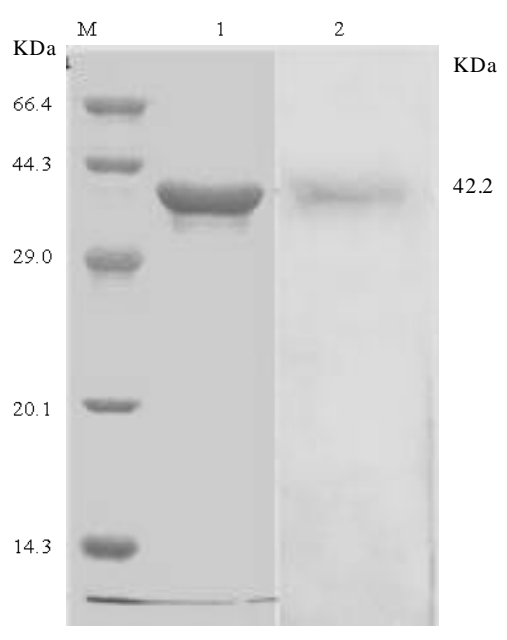

Fig. 3: Results of purification and western-blot of recombinant protein. Lane $\mathrm{M}$ : Protein marker. Lane 1: purified PET-32-ChIL-6. Lane 2: western-blot result of purified recombinant protein with pcDNA-ChIL-6 infected anti-sera

Table 1: ELISA detection of sera from mice immunized with pcDNA-ChI-6 plasmid (OD450 $\mathrm{nm}$ )

ELISA OD of Serum dilutions

values $(450 \mathrm{~nm})$

\begin{tabular}{lllllllll}
\hline Anti-pcDNA- & 1.541 & 1.422 & 1.215 & 1.037 & 0.998 & 0.831 & 0.548 & 0.396
\end{tabular}

ChIL-6 serum

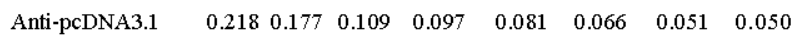

$(+)$ serum

Western blot analysis: The western blot analysis showed a strap of interest protein in the transformed membrane (Fig. 3), it suggested that the rPET-32-ChIL-6 can react positively with mouse anti-ChIL- 6 polyclonal antibody by immunoblot analysis.

Titer analysis by ELISA: About 20 control group mice sera were detected by ELISA, the average $\chi$ was 0.156 and Standard Deviation (SD) was 0.032 . The critical value was $\chi+3 \mathrm{SD}=0.252$. This means that when the $\mathrm{OD}$ value of sample $\geq 0.252$ and the ratio of positive sera and negative sera $(P / N) \geq 2.5$, the result was positive while the $O D$ value of sample $<0.252$, the result was negative.

The results of indirect ELISA experiment revealed that the mouse anti-ChIL- 6 polyclonal antibody could react positively with the rPET-32-ChIL-6 protein but serum from the mice immunized by anti-pcDNA3.1 $(+)$ react negatively with the rPET-32-ChIL-6 protein. The antibody titer of all the twelve sera could reached 1:5120 in ELISA and ELISA results of one representative positive serum and one negative serum were shown in Table 1. 


\section{DISCUSSION}

Interleukin-6 is an important cytokine mainly mediating humoral immunity but it's role in animal vaccination and infection is not clear now. It is reported that the biological activity of recombinant chicken IL-6 (rChIL-6) was similar to that of recombinant human IL-6 (rhuIL-6) with regard to in vitro proliferation of the IL-6-dependent murine hybridoma cell line 7TD1 and induced synthesis of corticosterone in vivo in chickens (Van Snick et al., 1986) this cross-species biological activity is an exception of cytokines in chicken (Xie et al., 2008). It had shown that rChIL-6 eukaryotic expression vector could improve the immune effect of LaSota vaccine of Newcastle disease vaccine significantly (Liu et al., 2009). IL-6 can be used as an indicator of immune status preparation of antibodies against chicken IL- 6 will make the basis for the development of serological method for the detection of chicken IL- 6 .

The comparison of published four sequences of chicken IL-6 on GenBank showed IL-6 gene of different kinds of chickens have high homology of no $<98 \%$ while having no significant similarity with those of the mammalian. In this study, IL-6 gene of chickens of Ya'an local species was cloned, it's nucleotide and amino acid sequence identities with other chicken IL-6 genes were $98-99 \%$. Bioinformatics analysis showed that this sequence contained 241 amino acid residues and had a long signal peptide sequences as mammals. The GC content of the DNA sequences of the signal peptides was high and reached $80 \%$.

In this study, we constructed a recombinant expressing plasmid pcDNA-ChIL- 6 by inserting the ChIL-6 gene into eukaryotic expression vector pcDNA3.1 $(+)$ under the control Cytomegalovirus (CMV) promoter, polyclonal antibodies was obtained by inoculating $\mathrm{BALB} / \mathrm{c}$ mouse. Then prokaryotic expression plasmid PET-ChIL-6 was constructed and expressed in E. coli BL21 effectively. The result of Western-blot and indirect ELISA showed that plasmid pcDNA-IL- 6 can be expressed in eukaryotic cells. The production of mouse anti-ChIL-6 polyclonal antibodies will help in developing a antigen-capture ELISA for the detection of chicken IL-6 which will be highly useful in deciphering the role of chicken IL-6 in immunological or pathological processes in chickens during vaccination or infections.

\section{CONCLUSION}

In conclusion, the polyclonal antibody against chicken Interleukin- 6 prepared by immunization of recombinant eukaryotic plasmid can react efficiently with its prokaryotic expression protein.

\section{ACKNOWLEDGEMENTS}

The research was financially supported by Program for Changjiang Scholars and Innovative Research Team in University PCSIRT (Grant No: IRTO848) and by Sichuan youth science and technology foundation (Grant number: 2007Q14-034)

\section{REFERENCES}

Gauldie, J., C. Richards, D. Harnish, P. Lansdorp and H. Baumann, 1987. Interferon beta 2/B-cell stimulatory factor type 2 shares identity with monocyte-derived hepatocyte-stimulating factor and regulates the major acute phase protein response in liver cells. Proc. Natl. Acad. Sci., 84: 7251-7255.

Hilton, L.S., A.G. Bean and J.W. Lowenthal, 2002. The emerging role of avian cytokines as immunotherapeutics and vaccine adjuvants. Vet. Immunol. Immunopathol., 85: 119-128.

Hirano, T., K. Yasukawa, H. Harada, T. Taga and Y. Watanabe et al., 1986. Complementary DNA for a novel human interleukin (BSF-2) that induces B lymphocytes to produce immunoglobulin. Nature, 324: 73-76.

Houssiau, F.A., P.G. Coulie, D. Olive and J. Van Snick, 1988. Synergistic activation of human T cells by interleukin 1 and interleukin 6. Eur. J. Immunol., 18: 653-656.

Ikebuchi, K., G.G. Wong, S.C. Clark, J.N. Thle, Y. Hirai and M. Ogawa, 1987. Interleukin 6 enhancement of interleukin 3-dependent proliferation of multipotential hemopoietic progenitors. Proc. Natl. Acad. Sci., 84: 9035-9039.

Karaca, K., J.M. Sharma, M.A. Tomai and R.L. Miller, 1996. In vivo and in vitro interferon induction in chickens by S-28828, an imidazoquinolinamine immunoenhancer. J. Interferon Cytokine Res., 164: $327-332$.

Kishimoto, T., S. Akira, M. Narazaki and T. Taga, 1995. Interleukin-6 family of cytokines and gp130. Blood, 86: 1243-1254.

Liu, R.L., N.L. Zou, H.N. Wang, P. Liu, Y. Huang, 2009. Construction of eukaryotic expression plasmids encoding chicken interleukin 26 and study on its immunoenhancement on newcastle disease lasota vaccine. Acta Vet. Zootechnica Sinica, 40: 93-97.

Muraguchi, A., T. Hirano, B. Tang, T. Matsuda, Y. Horii, K. Nakajima and T. Kishimoto, 1988. The essential role of B cell stimulatory factor 2 (BSF-2/L-6) for the terminal differentiation of B cells. J. Exp. Med., 167: 332-344. 
Poquan, J., 2001 . Cellular and Molecular Immunology. 5th Edn., Science Press, Beijing, China, ISBN: 9787 810860277.

Satoh, T., S. Nakamura, T. Taga, T. Matsuda, T. Hirano, T. Kishimoto and Y. Kaziro, 1988. Induction of neuronal differentiation in $\mathrm{PC} 12$ cells by B-cell stimulatory factor $2 /$ interleukin 6. Mol. Cell Biol., 8: 3546-3549.

Schneider, K., R. Klaas, B.Kaspers and P. Staeheli, 2001. Chicken interleukin-6: cDNA structure and biological properties. Eur. J. Biochem., 268: 4200-4206.

Sick, G., U. Schultz, U. Münster, J. Meier, B. Kaspers and P. Staeheli, 1998. Promoter structures and differential responses to viral and nonviral inducers of chicken type I interferongenes. J. Biol. Chem., 273: 9749-9754.

Van Snick, J., 1990. Interleukin-6: An overview. Annu. Rev. Immunol., 8: 253-278.
Van Snick, J., S. Cayphas, A. Vink, C. Uyttenhove, P.G. Coulie, M.R. Rubira and R.J. Simpson, 1986. Purification and NH2-terminal amino acid sequence of a T-cell-derived lymphokine with growth factor activity for B-cell hybridomas. Proc. Natl. Acad. Sci., 83: 9679-9683.

Xie, C.H., R.Q. Yan, B.A. Cui, X.L. Zhao, Z.M. Wu, Z.L. Zhang and J. Zhang, 2008. Enhancement of immune response to vaccines by recombinant chicken interleukin-6. Chinese J. Preventive Vet. Med., 30: 557-561.

Zou, N., F. Zhao, P. Liu, S. Cao, X. Wen and Y. Huang, 2010. The expression and characterization of highly antigenic region of spike protein of prevalent infectious bronchitis virus in Escherichia coli. J. Anim. Vet. Adv., 9: 1267-1274. 